\title{
Period Doublings in Coupled Parametrically Forced Damped Pendulums
}

\author{
Sang-Yoon Kim * \\ School of Physics, Georgia Institute of Technology, Atlanta, Georgia 30332-0430 \\ Kijin Lee \\ Department of Physics, Kangwon National University, Chunchon, Kangwon-Do 200-701, Korea
}

\begin{abstract}
We study period doublings in $N(N=2,3,4, \ldots)$ coupled parametrically forced damped pendulums by varying $A$ (the amplitude of the external driving force) and $c$ (the strength of coupling). With increasing $A$, the stationary point undergoes multiple period-doubling transitions to chaos. We first investigate the two-coupled case with $N=2$. For each period-doubling transition to chaos, the critical set consists of an infinity of critical line segments and the zero-coupling critical point lying on the line $A=A_{i}^{*}$ in the $A-c$ plane, where $A_{i}^{*}$ is the $i$ th transition point for the uncoupled case. We find three kinds of critical behaviors, depending on the position on the critical set. They are the same as those for the coupled one-dimensional maps. Finally, the results of the $N=2$ case are extended to many-coupled cases with $N \geq 3$, in which the critical behaviors depend on the range of coupling.
\end{abstract}

PACS numbers: 05.45.+b, 03.20.+i, 05.70.Jk

Typeset using REVTEX

*Permanent address: Department of Physics, Kangwon National University, Chunchon, KangwonDo 200-701, Korea. Electronic address: sykim@cc.kangwon.ac.kr 


\section{INTRODUCTION}

In recent years, much attention has been paid to coupled nonlinear oscillators. Such coupled oscillators are used to model many physical, chemical, and biological systems such as coupled p-n junctions [1], Josephson-junction arrays [2], the charge-density waves [3], chemical-reaction systems [4], and biological-oscillation systems [5]. They are known to exhibit period-doubling bifurcations (PDB's), saddle-node bifurcations, Hopf bifurcations, chaos as well as pattern formation.

The coupled oscillators investigated in this paper are coupled parametrically forced damped pendulums (PFDP's). For a single damped pendulum, vertical oscillation of its support leads to a time-periodic variation of its natural frequency, and hence it is called a PFDP [6,7]. This simple PFDP shows richness in its dynamical behavior [8 10]. One of the interesting behaviors is that with increasing the amplitude $A$ of the vertical oscillation, the stationary point undergoes multiple period-doubling transitions to chaos, which have been found in our recent work [1].

Here we study the critical behaviors of PDB's in $N(N=2,3,4, \ldots)$ coupled PFDP's. The "coupling effect" of the nature, strength, and range of coupling on the critical behaviors are particularly investigated. The coupled PFDP's exhibit multiple period-doubling transitions to chaos. This is in contrast to the case of the coupled one-dimensional (1D) maps, in which only single period-doubling transition to chaos occurs [12,13. We first consider the simplest coupled case with $N=2$. For each period-doubling transition to chaos, the critical set (set of the critical points) is composed of the zero-coupling critical point and an infinity of critical line segments lying on the line $A=A_{i}^{*}$ in the $A-c$ plane, where $A$ is the amplitude of the external driving force, $c$ a coupling parameter, and $A_{i}^{*}$ the $i$ th perioddoubling transition point for the uncoupled case. It is found that there exist three kinds of critical behaviors, depending on the position on the critical set. These critical behaviors are the same as those of the coupled one-dimensional (1D) maps found by one of us and Kook [13. The results for the two-coupled case are also extended to many-coupled cases with 
$N \geq 3$. It is found that the critical behaviors for the many-coupled cases vary depending on whether or not the coupling is global. In the extreme long-range case of global coupling, in which each PFDP is coupled to all the other ones with equal coupling strength, the critical behaviors are the same as those for the two-coupled case, irrespectively of $N$. However, for any other nonglobal-coupling cases of shorter-range couplings, an important change occurs in the stability diagram of $2^{n}$-periodic $(n=0,1,2, \ldots)$ orbits in the $A-c$ plane, and consequently the structure of the critical set becomes different from that for the global-coupling case. To the best of our knowledge, so far only the authors of the paper [14] attempted to study the critical behaviors of PDB's in coupled oscillators. However, only the critical behaviors near the zero-coupling critical point were considered, because the existence of an infinity of additional critical line segments in the coupled 1D maps was not known at that time. Moreover, any explicit numerical values of the scaling factors for the zero-coupling case were not obtained [15].

This paper is organized as follows. We first introduce two coupled PFDP's and discuss their general properties in Sec. III. Stability of periodic orbits, bifurcations, and Lyapunov exponents are also discussed there. In Sec. III, the critical behaviors of PDB's for the twocoupled case are studied by varying two parameters $A$ and $c$. We also extend the results of the two-coupled case to many coupled PFDP's in Sec. IV. Finally, a summary is given in Sec. V.

\section{STABILITY OF PERIODIC ORBITS, BIFURCATIONS, AND LYAPUNOV EXPONENTS IN TWO COUPLED PFDP'S}

In this section, we first discuss stability of period orbits in the Poincaré map of the two coupled PFDP's, using the Floquet theory. Bifurcations associated with the stability and Lyapunov exponents are then discussed.

Consider a system consisting of two identical PFDP's coupled symmetrically:

$$
\ddot{x}_{1}=f\left(x_{1}, \dot{x}_{1}, t\right)+g\left(x_{1}, x_{2}\right),
$$




$$
\ddot{x}_{2}=f\left(x_{2}, \dot{x}_{2}, t\right)+g\left(x_{2}, x_{1}\right) .
$$

where

$$
f(x, \dot{x}, t)=-2 \pi \gamma \dot{x}-2 \pi\left(\omega_{0}^{2}-A \cos 2 \pi t\right) \sin 2 \pi x
$$

and $g\left(x_{1}, x_{2}\right)$ is a coupling function, obeying the condition $g(x, x)=0$ for all $x$. Here $x$ is the angular position, $\gamma$ the damping coefficient, $\omega_{0}$ the natural frequency of the pendulum, $A$ the amplitude of the external driving force of period one, and we consider the coupling function $g\left(x_{1}, x_{2}\right)$ of the form,

$$
g\left(x_{1}, x_{2}\right)=\frac{c}{2}\left[u\left(x_{2}\right)-u\left(x_{1}\right)\right]
$$

where $u(x)$ is a function of one variable, and $c$ a coupling parameter.

The two second-order ordinary differential equations (11) are reduced to four first-order ordinary differential equations:

$$
\begin{aligned}
& \dot{x}_{1}=y_{1}, \\
& \dot{y}_{1}=f\left(x_{1}, y_{1}, t\right)+g\left(x_{1}, x_{2}\right), \\
& \dot{x}_{2}=y_{2}, \\
& \dot{y}_{2}=f\left(x_{2}, y_{2}, t\right)+g\left(x_{2}, x_{1}\right) .
\end{aligned}
$$

Consider an initial point $\mathbf{z}(0)\left[\equiv\left(z_{1}(0), z_{2}(0)\right)\right]$, where $z_{i}=\left(x_{i}, y_{i}\right)(i=1,2)$. Then, its Poincaré maps can be computed by sampling the points $\mathbf{z}(m)$ at the discrete time $m$, where $m=1,2,3, \ldots$. We call the transformation $\mathbf{z}(m) \rightarrow \mathbf{z}(m+1)$ the Poincaré (time-1) map, and write $\mathbf{z}(m+1)=P(\mathbf{z}(m))$.

The four-dimensional (4D) Poincaré map $P$ has an exchange symmetry such that

$$
S_{1} P S_{1}(\mathbf{z})=P(\mathbf{z}) \text { for all } \mathbf{z}
$$

where $S_{1}\left(z_{1}, z_{2}\right)=\left(z_{2}, z_{1}\right)$. The set of all points, which are invariant under the exchange of coordinates $S_{1}$, forms a synchronous plane on which $x_{1}=x_{2}$ and $y_{1}=y_{2}$. An orbit is called a(n) (in-phase) synchronous orbit if it lies on the synchronous plane, i.e., it satisfies 


$$
x_{1}(m)=x_{2}(m) \equiv x^{*}(m), \quad y_{1}(m)=y_{2}(m) \equiv y^{*}(m) \text { for all } m
$$

Otherwise, it is called an (out-of-phase) asynchronous orbit. Here we study only the synchronous orbits. They can be easily found from the uncoupled PFDP, because the coupling function satisfies $g\left(x^{*}, x^{*}\right)=0$. Note also that for the cases of these synchronous orbits, the 4D Poincaré map $P$ also has the inversion symmetry such that

$$
S_{2} P S_{2}(\mathbf{z})=P(\mathbf{z}) \text { for all } \mathbf{z}
$$

where $S_{2}(\mathbf{z})=-\mathbf{z}$. If a synchronous orbit $\{\mathbf{z}(m)\}$ of $P$ is invariant under $S_{2}$, it is called a symmetric orbit. Otherwise, it is called an asymmetric orbit and has its "conjugate" orbits $S_{2}\{\mathbf{z}(m)\}$.

We now study the stability of a synchronous periodic orbit with period $q$ such that $P^{q}(\mathbf{z}(0))=\mathbf{z}(0)$ but $P^{j}(\mathbf{z}(0)) \neq \mathbf{z}(0)$ for $1 \leq j \leq q-1$. Here $P^{k}$ means the $k$-times iterated map. The linear stability of the $q$-periodic orbit is determined from the linearizedmap matrix $D P^{q}(\mathbf{z}(0))$ of $P^{q}$ at an orbit point $\mathbf{z}(0)$. Using the Floquet theory [16], the matrix $D P^{q}$ can be obtained by integrating the linearized differential equations for small perturbations as follows.

Stability analysis of an orbit can be conveniently carried out in a set of new coordinates $\left(X_{1}, Y_{1}, X_{2}, Y_{2}\right)$ defined by

$$
\begin{aligned}
& X_{1}=\frac{\left(x_{1}+x_{2}\right)}{2}, Y_{1}=\frac{\left(y_{1}+y_{2}\right)}{2}, \\
& X_{2}=\frac{\left(x_{1}-x_{2}\right)}{2}, Y_{2}=\frac{\left(y_{1}-y_{2}\right)}{2} .
\end{aligned}
$$

Here the first and second pairs of coordinates $Z_{1}$ and $Z_{2}$, defined by $Z_{i} \equiv\left(X_{i}, Y_{i}\right)(i=1,2)$, correspond to the synchronous and asynchronous modes of the orbit, respectively. For example, for a synchronous orbit $Z_{1}=\left(x^{*}, y^{*}\right)$ and $Z_{2}=(0,0)$, while for an asynchronous orbit, $Z_{2} \neq(0,0)$. Hereafter, we will call $Z_{1}$ and $Z_{2}$ the synchronous and asynchronous modes of the orbit, respectively.

Let $\mathbf{Z}(t)\left[\equiv\left(Z_{1}, Z_{2}\right)\right.$ ] be a solution lying on the closed orbit corresponding to a synchronous $q$-periodic orbit with $\mathbf{Z}(t)=\mathbf{Z}(t+q)$. In order to study the stability of the 
synchronous closed orbit, we consider an infinitesimal perturbation $\delta \mathbf{Z}\left[\equiv\left(\delta Z_{1}, \delta Z_{2}\right)\right]$ to the orbit. Note that $\delta Z_{1}$ and $\delta Z_{2}$ are the synchronous and asynchronous modes of the perturbation to the synchronous orbit, respectively. Linearizing the ordinary differential equations (四) (expressed in terms of the new coordinates) about the orbit, we obtain

$$
\left(\begin{array}{c}
\delta \dot{Z}_{1} \\
\delta \dot{Z}_{2}
\end{array}\right)=J(t)\left(\begin{array}{c}
\delta Z_{1} \\
\delta Z_{2}
\end{array}\right)
$$

where

$$
J=\left(\begin{array}{cc}
J_{1} & 0 \\
0 & J_{2}
\end{array}\right)
$$

Here $\mathbf{0}$ is the $2 \times 2$ null matrix, and

$$
\begin{aligned}
& J_{1}(t)=\left(\begin{array}{cc}
0 & 1 \\
f_{1}\left(x^{*}, \dot{x}^{*}, t\right) & f_{2}\left(x^{*}, \dot{x}^{*}, t\right)
\end{array}\right), \\
& J_{2}(t)=\left(\begin{array}{cc}
0 & 1 \\
f_{1}\left(x^{*}, \dot{x}^{*}, t\right)-2 G\left(x^{*}\right) & f_{2}\left(x^{*}, \dot{x}^{*}, t\right)
\end{array}\right),
\end{aligned}
$$

where

$$
\begin{aligned}
& f_{1}\left(x^{*}, \dot{x}^{*}, t\right) \equiv \frac{\partial f\left(x^{*}, \dot{x}^{*}, t\right)}{\partial x^{*}}=-4 \pi^{2}\left(\omega_{0}^{2}-A \cos 2 \pi t\right) \cos 2 \pi x^{*}(t), \\
& f_{2}\left(x^{*}, \dot{x}^{*}, t\right) \equiv \frac{\partial f\left(x^{*}, \dot{x}^{*}, t\right)}{\partial \dot{x}^{*}}=-2 \pi \gamma
\end{aligned}
$$

and

$$
\left.G\left(x^{*}\right) \equiv \frac{\partial g\left(x_{1}, x_{2}\right)}{\partial x_{2}}\right|_{x_{1}=x_{2}=x^{*}}=\frac{c}{2} u^{\prime}\left(x^{*}\right) .
$$

Here the prime denotes the differentiation.

Since the $4 \times 4$ matrix $J$ of Eq. (10) is decomposed into two $2 \times 2$ submatrices $J_{1}$ and $J_{2}$, Eq. (9) is reduced to two independent equations,

$$
\delta \dot{Z}_{i}=J_{i}(t) \delta Z_{i} \text { for } i=1,2 .
$$


That is, $\delta Z_{1}$ (synchronous-mode perturbation) and $\delta Z_{2}$ (asynchronous-mode perturbation) become decoupled for the case of a synchronous orbit. Note also that each $J_{i}(i=1,2)$ is a $q$-periodic matrix. Let $W_{i}(t)=\left(w_{i}^{(1)}(t), w_{i}^{(2)}(t)\right)$ be a fundamental solution matrix with $W_{i}(0)=I$. Here $w_{i}^{(1)}(t)$ and $w_{i}^{(2)}(t)$ are two independent solutions expressed in column vector forms, and $I$ is the $2 \times 2$ unit matrix. Then a general solution of the $q$-periodic system has the following form

$$
\delta Z_{i}(t)=W_{i}(t) \delta Z_{i}(0), W_{i}(0)=I
$$

Substitution of Eq. (17) into Eq. (16) leads to an initial-value problem to determine $W_{i}(t)$,

$$
\dot{W}_{i}(t)=J_{i}(t) W_{i}(t), W_{i}(0)=I .
$$

In this system of new coordinates, the linearized-map matrix $M\left(\equiv D P^{q}\right)$ has the following block-diagonalized form,

$$
M=\left(\begin{array}{cc}
M_{1} & \mathbf{0} \\
\mathbf{0} & M_{2}
\end{array}\right),
$$

where each $2 \times 2$ submatrix $M_{i}\left[\equiv W_{i}(q)\right](i=1,2)$ is calculated through integration of Eq. (18) over the period $q$. In order to determine the eigenvalues of $M$, it is sufficient to solve the eigenvalue problems for the two submatrices $M_{1}$ and $M_{2}$, independently. Here the submatrices $M_{1}$ and $M_{2}$ determine the stability of the synchronous orbit against the synchronous-mode and asynchronous-mode perturbations, respectively. Note also that the first submatrix $M_{1}$ is just the linearized Poincaré map of the PFDP [11, and the coupling affects only the second submatrix $M_{2}$.

The characteristic equation of each submatrix $M_{i}(i=1,2)$ is

$$
\lambda_{i}^{2}-\operatorname{tr} M_{i} \lambda_{i}+\operatorname{det} M_{i}=0
$$

where $\operatorname{tr} M_{i}$ and $\operatorname{det} M_{i}$ denote the trace and determinant of $M_{i}$, respectively. As shown in [17], det $M_{i}$ is calculated from a formula 


$$
\operatorname{det} M_{i}=e^{\int_{0}^{q} \operatorname{tr} J_{i} d t}
$$

Substituting the trace of $J_{i}$ (i.e., $\operatorname{tr} J_{i}=-2 \pi \gamma$ ) into Eq. (21), we obtain

$$
\operatorname{det} M_{1}=\operatorname{det} M_{2}=e^{-2 \pi \gamma q} \text {. }
$$

Hence, both the submatrices $M_{1}$ and $M_{2}$ have the same constant Jacobian determinant (less than unity). The eigenvalues, $\lambda_{i, 1}$ and $\lambda_{i, 2}$, of $M_{i}$ are called the Floquet stability multipliers of the synchronous $q$-periodic orbit. The first (second) pair of stability multipliers $\left(\lambda_{1,1}, \lambda_{1,2}\right)\left[\left(\lambda_{2,1}, \lambda_{2,2}\right)\right]$ of $M_{1}\left(M_{2}\right)$ is associated with stability against the synchronous-mode (asynchronous-mode) perturbation, and hence it may be called the pair of synchronous (asynchronous) stability multipliers. Note also that the pair of synchronous stability multipliers is just the pair of stability multipliers of the uncoupled PFDP [11], and the coupling affects only the pair of asynchronous stability multipliers.

Each pair of stability multipliers $\left(\lambda_{i, 1}, \lambda_{i, 2}\right)(i=1,2)$ lies either on the circle of radius $e^{-\pi \gamma q}$, or on the real axis in the complex plane. The synchronous orbit is stable when it is stable against both the synchronous-mode and asynchronous-mode perturbations, i.e., the moduli of all its four stability multipliers are less than unity. We first note that all the stability multipliers never cross the unit circle in the complex plane, and hence Hopf bifurcations do not occur. Consequently, the synchronous orbit can lose its stability only when a multiplier decreases (increases) through -1 (1) on the real axis.

Associate with each pair of stability multipliers $\left(\lambda_{i, 1}, \lambda_{i, 2}\right)$ a quantity $R_{i}$, called the residue,

$$
R_{i} \equiv \frac{1+\operatorname{det} M_{i}-\operatorname{tr} M_{i}}{2\left(1+\operatorname{det} M_{i}\right)}
$$

which was introduced in [18] to characterize stability of periodic orbits in 2D dissipative maps with constant Jacobian determinants. Here the first and second residues $R_{1}$ and $R_{2}$ are associated with stability of the synchronous orbit against the synchronous-mode and asynchronous-mode perturbations, respectively. Hereafter, they will be called the synchronous and asynchronous residues, respectively. Note also that the synchronous residue 
$R_{1}$ is just the residue of the uncoupled PFDP [11], and the coupling affects only the asynchronous residue $R_{2}$. A synchronous periodic orbit is stable when $0<R_{i}<1$ for $i=1$, 2; at both ends of $R_{i}=0$ and 1 , the stability multipliers $\lambda_{i}$ 's are 1 and -1 , respectively. When each residue $R_{i}$ decreases through 0 (i.e., $\lambda_{i}$ increases through 1 ), the periodic orbit loses its stability via saddle-noddle or pitchfork or transcritical bifurcation. On the other hand, when $R_{i}$ increases through 1 (i.e., $\lambda_{i}$ decreases through -1 ), it becomes unstable via PDB, also referred to as a flip or subharmonic bifurcation. For each case of the PDB's and the pitchfork bifurcations (PFB's), two types of supercritical and subcritical bifurcations occur. (For more details on bifurcations in 2D dissipative maps, refer to Ref. 19. .)

The stable region of a synchronous periodic orbit in the $A-c$ plane is bounded by bifurcation lines associated with PDB's and PFB's (i.e., those curves determined by the equations $R_{i}=0$ and 1 for $i=1,2$ ), as will be seen in Sec. [II]. When the boundary lines on which $R_{1}\left(R_{2}\right)=0$ and 1 are crossed, the synchronous orbit loses its stability via synchronous (asynchronous) PFB and PDB, respectively. For each case of the synchronous (asynchronous) PFB and PDB, two types of supercritical and subcritical bifurcations take place. In the supercritical case of the synchronous (asynchronous) PFB and PDB, the synchronous orbit loses its stability, and gives rise to the birth of a pair of new stable synchronous (asynchronous) orbits with the same period and a new stable synchronous (asynchronous) period-doubled orbit, respectively. However, in the subcritical case of the synchronous (asynchronous) PFB and PDB, the synchronous orbit becomes unstable by absorbing a pair of unstable synchronous (asynchronous) orbits with the same period and an unstable synchronous (asynchronous) period-doubled orbit, respectively.

Finally, we briefly discuss Lyapunov exponents of a synchronous orbit in the Poincaré map $P$, characterizing the mean exponential rate of divergence of nearby orbits [20]. The synchronous and asynchronous modes of a nearby orbit are decoupled, because the linearized Poincaré map $D P$ at the synchronous orbit is just the block-diagonalized matirx $M$ of Eq. (19) with $q=1$. Therefore, the $2 \times 2$ submatrices $M_{1}$ and $M_{2}$ of $M$ determine the pairs of synchronous and asynchronous Lyapunov exponents $\left(\sigma_{1,1}, \sigma_{1,2}\right)$ and $\left(\sigma_{2,1}, \sigma_{2,2}\right)$, characterizing 
the average exponential rate of divergence of the synchronous and asynchronous modes of a nearby orbit, respectively, where $\sigma_{i, 1} \geq \sigma_{i, 2}$ for $i=1,2$. Since the two submatrices have the same constant Jacobian determinant of Eq. (22), each pair of the Lyapunov exponents satisfy $\sigma_{i, 1}+\sigma_{i, 2}=-2 \pi \gamma(i=1,2)$. Note also that the first pair of synchronous Lyapunov exponents $\left(\sigma_{1,1}, \sigma_{1,2}\right)$ is just the pair of the Lyapunov exponents of the uncoupled PFDP, and the coupling affects only the second pair of asynchronous Lyapunov exponents $\left(\sigma_{2,1}, \sigma_{2,2}\right)$.

\section{CRITICAL BEHAVIORS IN TWO COUPLED PFDP'S}

In this section, by varying the two parameters $A$ and $c$, we study the critical behaviors of the synchronous supercritical PDB's in the two coupled PFDP's (1) for $\gamma=0.1$ and $\omega_{0}=0.5$. The two coupled PFDP's exhibit multiple period-doubling transitions to chaos, which is in contrast to the case of the coupled 1D maps with only single period-doubling transition to chaos 12,13. For each transition to chaos, the zero-coupling critical point and an infinity of critical line segments constitute the critical set in the $A-c$ plane. There exist three kinds of critical behaviors, depending on the position on the critical set. These critical behaviors are found to be the same as those of the two coupled 1D maps [13].

We consider a linearly-coupled case in which the coupling function (3) is

$$
g\left(x_{1}, x_{2}\right)=\frac{c}{2}\left(x_{2}-x_{1}\right)
$$

Figure 1 shows the stability diagram of the synchronous orbits with low period $q=1,2$. The stable region of a synchronous orbit is bounded by its PDB and PFB lines. The horizontal (non-horizontal) solid and short-dashed boundary lines correspond to synchronous (asynchronous) PDB and PFB lines, respectively. (Each bifurcation may be supercritical or subcritical.) Note also that the horizontal synchronous PDB or PFB lines extend to the (plus) infinity $(c=\infty)$. For the sake of convenience, only some parts (up to $c=7$ ) of the infinitely long lines are drawn in the figure.

We first consider the bifurcations associated with stability of the stationary point $\left[\left(x_{1}, y_{1}, x_{2}, y_{2}\right)=(0,0,0,0)\right]$. Its stable region is denoted by $\mathrm{SP}$ in Fig. 11. When the non- 
horizontal short-dashed line of the SP is crossed, the stationary point becomes unstable via asynchronous subcritical PFB. However, at the horizontal solid boundary line, it loses its stability via synchronous supercritical PDB, and a new stable synchronous orbit of period 2 appears. The 2-periodic orbit is a symmetric orbit with respect to the inversion symmetry $S_{2}$, and its stable region is denoted by SP2 in Fig. 1. When the horizontal (non-horizontal) shortdashed boundary line of the SP2 is crossed, the symmetric 2-periodic orbit loses its stability via synchronous (asynchronous) supercritical PFB, and a pair of new stable synchronous (asynchronous) orbits with period 2 appears. Note that the new pair of synchronous orbits is a conjugate pair of asymmteric orbits with respect to the $S_{2}$-symmetry. Its stable region is denoted by ASP2 in Fig. 1. Unlike the cases of the lower-level stability regions (SP and SP2), the ASP2 is $U$-shaped, because a parabolalike asynchronous PDB line also is a boundary line of the ASP2. An asynchronous supercritical PDB occurs at the parabolalike solid line, whereas an asynchronous subcritical PFB takes place at the non-horizontal shortdashed line. However, each synchronous asymmetric 2-periodic orbit becomes unstable via synchronous supercritical PDB when the horizontal solid line is crossed, and gives rise to the birth of a new synchronous asymmetric 4-periodic orbit. Here we are interested in such synchronous supercritical PDB's.

Figure 2 shows the stability diagram of synchronous asymmetric orbits born by synchronous supercritical PDB's. Each synchronous asymmetric orbit of level $n$ (period $2^{n}$, $n=1,2,3, \ldots)$ loses its stability at the horizontal solid line of its stable region via synchronous supercritical PDB, and gives rise to the birth of a synchronous asymmetric period-doubled orbit of level $n+1$. Such an infinite sequence ends at a finite value of $A_{1}^{*}=0.3577098453$, which is the first period-doubling transition point of the uncoupled PFDP [11]. Consequently, a synchronous quasiperiodic orbit, whose maximum synchronous Lyapunov exponent is zero (i.e., $\sigma_{1,1}=0$ ), exists on the $A=A_{1}^{*}$ line.

We examine the treelike structure of the stability diagram in Fig. 2, which consists of an infinite pile of $U$-shape regions and rectangular-shape regions. Note that the treelike structure is asymptotically the same as that in the coupled 1D maps [13]. The $U$-shape 
branching is repeated at one side of each $U$-shape region, including the $c=0$ line segment. The branching side will be referred to as the zero $c$ side. However, the other side of each $U$-shape region grows like a chimney without any further branchings [as an example, see the branch starting from the right side of the ASP2 in Fig. 2(b)]. As in the coupled 1D maps [13], this rule governs the asymptotic behavior of the treelike structure, even though there are a few exceptions for lower-level orbits. Other type of $U$-shape regions without the zero $c$ sides [e.g., the leftmost $U$-shape region in the third-level stability region in Fig. 2(a)] may appear in the lower-level stability regions. However, the $U$-shape branching for this kind of $U$-shape region ends at some finite level, and then each side of the $U$-shape region grows like a chimney without any further branchings. Consequently, an infinite number of successive branchings occur only for the case of the $U$-shape region with the zero $c$ side.

A sequence of connected stability regions with increasing period is called a "perioddoubling route" [13]. There are two kinds of period-doubling routes. The sequence of the $U$-shape regions with the zero $c$ sides converges to the zero-coupling point $c=0$ on the $A=A_{1}^{*}$ line. It will be referred to as the $U$ route. On the other hand, a sequence of rectangular regions in each chimney converges to a critical line segment on the $A=A_{1}^{*}$ line. For examples, the rightmost one in Fig. 2(a) is the line segment joining the left end point $c_{l}(=0.343687 \cdots)$ and the right end point $c_{r}(=0.484777 \cdots)$ on the $A=A_{1}^{*}$ line, and the one in Fig. 2(b) is the infinitely-long line connecting the two end points $c_{l}(=4.407457 \cdots)$ and $c_{r}(=\infty)$ on the $A=A_{1}^{*}$ line. This kind of route will be called a $C$ route. Note that there are infinitely many $C$ routes, while the $U$ route converging to the zero-coupling critical point $\left(A_{1}^{*}, 0\right)$ is unique. Hence, an infinite number of critical line segments, together with the zero-coupling critical point, constitute the critical set.

We now study the critical behaviors on the critical set. First, consider the case of the $U$ route ending at the zero-coupling critical point. We follow the synchronous orbits of period $q=2^{n}$ up to level $n=9$ in the $U$ route, and obtain a self-similar sequence of parameters $\left(A_{n}, c_{n}\right)$, at which each orbit of level $n$ has some given residues $R_{1}$ and $R_{2}$ (e.g., $R_{1}=1$ and $\left.R_{2}=0\right)$. Then the sequence $\left\{\left(A_{n}, c_{n}\right)\right\}$ converges geometrically to the zero-coupling critical 
point $\left(A_{1}^{*}, 0\right)$. In order to see the convergence of each of the two scalar sequences $\left\{A_{n}\right\}$ and $\left\{c_{n}\right\}$, we define

$$
\delta_{n} \equiv \frac{\Delta A_{n}}{\Delta A_{n+1}}, \quad \mu_{n} \equiv \frac{\Delta c_{n}}{\Delta c_{n+1}}
$$

where $\Delta A_{n}=A_{n}-A_{n-1}$ and $\Delta c_{n}=c_{n}-c_{n-1}$. The sequences of $\delta_{n}$ and $\mu_{n}$ are listed in Table $\llbracket$, and converge to their limit values, $\delta(\simeq 4.67)$ and $\mu(\simeq-2.5)$, respectively. Hence the two sequences $\left\{A_{n}\right\}$ and $\left\{c_{n}\right\}$ obey one-term scaling laws asymptotically:

$$
\Delta A_{n} \sim \delta^{-n}, \quad \Delta c_{n} \sim \mu^{-n} \text { for large } n .
$$

As in the coupled 1D maps, the value of the nonlinearity-parameter scaling factor $\delta$ agrees well with the Feigenbaum constant $(=4.669 \cdots)$ of the 1D maps [21]. The value of the coupling-parameter scaling factor $\mu$ is also close to that $(=-2.502 \cdots)$ of the couplingparameter scaling factor $\alpha$ of the coupled 1D maps near the zero-coupling critical point [13. It has been also shown in [13] that the scaling factor $\alpha$ is just the first relevant "coupling eigenvalue" (CE) $\nu_{1}$ of the zero-coupling fixed map of the renormalization transformation for the case of the coupled 1D maps. In addition to $\nu_{1}=\alpha$, the zero-coupling fixed map has another second relevant $\mathrm{CE} \nu_{2}(=2)$, which also affects the scaling associated with coupling in the coupled 1D map [22].

In order to get a correction to the leading scaling (26), we take into account the effect of the second relevant $\mathrm{CE} \nu_{2}(=2)$ on the scaling of the sequence $\left\{\Delta c_{n}\right\}$ and extend the simple one-term scaling law (26) to a two-term scaling law 22, 23:

$$
\Delta c_{n} \sim C_{1} \mu_{1}^{-n}+C_{2} \mu_{2}^{-n} \text { for large } n
$$

where $\left|\mu_{2}\right|>\left|\mu_{1}\right|$, and $C_{1}$ and $C_{2}$ are some constants. This is a kind of multiple scaling law [24]. Eq. (27) gives

$$
\Delta c_{n}=s_{1} \Delta c_{n+1}-s_{2} \Delta c_{n+2},
$$

where $s_{1}=\mu_{1}+\mu_{2}$ and $s_{2}=\mu_{1} \mu_{2}$. Then, $\mu_{1}$ and $\mu_{2}$ are solutions of the following quadratic equation, 


$$
\mu^{2}-s_{1} \mu+s_{2}=0
$$

To evaluate $\mu_{1}$ and $\mu_{2}$, we first obtain $s_{1}$ and $s_{2}$ from $\Delta c_{n}$ 's using Eq. (28):

$$
s_{1}=\frac{\Delta c_{n} \Delta c_{n+1}-\Delta c_{n-1} \Delta c_{n+2}}{\Delta c_{n+1}^{2}-\Delta c_{n} \Delta c_{n+2}}, \quad s_{2}=\frac{\Delta c_{n}^{2}-\Delta c_{n+1} \Delta c_{n-1}}{\Delta c_{n+1}^{2}-\Delta c_{n} \Delta c_{n+2}} .
$$

Note that Eqs. (27)-(30) hold only for large $n$. In fact, the values of $s_{i}$ 's and $\mu_{i}{ }^{\prime}$ 's $(i=1,2)$ depend on the level $n$. Therefore, we explicitly denote $s_{i}$ 's and $\mu_{i}$ 's by $s_{i, n}$ 's and $\mu_{i, n}$ 's, respectively. Then, each of them converges to a constant as $n \rightarrow \infty$ :

$$
\lim _{n \rightarrow \infty} s_{i, n}=s_{i}, \quad \lim _{n \rightarrow \infty} \mu_{i, n}=\mu_{i}, \quad i=1,2 .
$$

Three sequences $\left\{\mu_{1, n}\right\},\left\{\mu_{2, n}\right\}$, and $\left\{\mu_{1, n}^{2} / \mu_{2, n}\right\}$ are shown in Table II. The second column shows rapid convergence of the first scaling factor $\mu_{1, n}$ to its limit value $\mu_{1}(\simeq-2.50)$, which agrees well with the first relevant $\mathrm{CE} \nu_{1}(=\alpha)$. (Its convergence to $\alpha$ is faster than that for the case of the above one-term scaling law.) The second scaling factor $\mu_{2, n}$ also seems to converge slowly to its limit value $\mu(\simeq 3.1)$, whose accuracy is lower than that of $\mu_{1}$. As in the coupled area-preserving maps [23], it seems from the third and fourth columns that the second scaling factor $\mu_{2}$ may be expressed by a product of two relevant CE's $\nu_{1}(=\alpha)$ and $\nu_{2}(=2)$,

$$
\mu_{2}=\frac{\nu_{1}^{2}}{\nu_{2}}
$$

It has been known that every scaling factor in the multiple-scaling expansion of a parameter is expressed by a product of the eigenvalues of a linearized renormalization operator [24].

We also study the coupling effect on the asynchronous residue $R_{2, n}$ of the synchronous orbit of period $2^{n}$ near the zero-coupling critical point $\left(A_{1}^{*}, 0\right)$. Figure 3 shows three plots of $R_{2, n}\left(A_{1}^{*}, c\right)$ versus $c$ for $n=4,5$, and 6 . For $c=0, R_{2, n}$ converges to a constant $R_{2}^{*}$ $(=1.30059 \ldots)$, called the critical asynchronous residue, as $n \rightarrow \infty$. However, when $c$ is nonzero $R_{2, n}$ diverges as $n \rightarrow \infty$, i.e., its slope $S_{n}\left(\left.\equiv \frac{\partial R_{2, n}}{\partial c}\right|_{\left(a^{*}, 0\right)}\right)$ at the zero-coupling critical point diverges as $n \rightarrow \infty$. 
As in the coupled area-preserving maps [23], the sequence $\left\{S_{n}\right\}$ also obeys a two-term scaling law,

$$
S_{n}=D_{1} \nu_{1}^{n}+D_{2} \nu_{2}^{n} \quad \text { for large } n
$$

where $\left|\nu_{1}\right|>\left|\nu_{2}\right|$. This equation gives

$$
S_{n+2}=r_{1} S_{n+1}-r_{2} S_{n}
$$

where $r_{1}=\nu_{1}+\nu_{2}$ and $r_{2}=\nu_{1} \nu_{2}$. As in the scaling for the coupling parameter, we first obtain $r_{1}$ and $r_{2}$ of level $n$ from $S_{n}$ 's:

$$
r_{1, n}=\frac{S_{n+1} S_{n}-S_{n+2} S_{n-1}}{S_{n}^{2}-S_{n+1} S_{n-1}}, \quad r_{2, n}=\frac{S_{n+1}^{2}-S_{n} S_{n+2}}{S_{n}^{2}-S_{n+1} S_{n-1}} .
$$

Then, the scaling factors $\nu_{1, n}$ and $\nu_{2, n}$ of level $n$ are given by the roots of the quadratic equation,

$$
\nu_{n}^{2}-r_{1, n} \nu_{n}+r_{2, n}=0
$$

They are listed in Table III and converge to constants $\nu_{1}(\simeq-2.503)$ and $\nu_{2}(\simeq 2)$ as $n \rightarrow \infty$, whose accuracies are higher than those of the coupling-parameter scaling factors. Note that the values of $\nu_{1}$ and $\nu_{2}$ agree well with those of the two relevant CE's $\nu_{1}$ and $\nu_{2}$.

We next consider the cases of $C$ routes, each of which converges to a critical line segment. Two kinds of additional critical behaviors are found at each critical line segment; the one critical behavior exists at both ends and the other critical behavior exists at interior points. In each $C$ route, there are two kinds of self-similar sequences of parameters $\left(A_{n}, c_{n}\right)$, at which each synchronous orbit of level $n$ has some given residues $R_{1}$ and $R_{2}$; the one converges to the left end point of the critical line segment and the other converges to the right end point. As an example, consider the rightmost $C$ route in Fig. \&(a), which converges to the critical line segment with two ends $\left(A_{1}^{*}, c_{l}\right)$ and $\left(A_{1}^{*}, c_{r}\right)$. We follow, in the rightmost $C$ route, two selfsimilar sequences of parameters, one converging to the left end and the other converging to the right end. In both cases, the sequence $\left\{A_{n}\right\}$ converges geometrically to its accumulation value $A_{1}^{*}$ with the $1 \mathrm{D}$ scaling factor $\delta(\simeq 4.67)$ like the case of the $U$ route, 


$$
\Delta A_{n} \sim \delta^{-n} \text { for large } n,
$$

where $\Delta A_{n}=A_{n}-A_{n-1}$. The sequences $\left\{c_{n}\right\}$ for both cases also obey the one-term scaling law,

$$
\Delta c_{n} \sim \mu^{-n} \text { for large } n
$$

where $\Delta c_{n}=c_{n}-c_{n-1}$. The sequence of the scaling factor $\mu_{n}$ of level $n$ is listed in Table 『, and converges to its limit value $\mu(\simeq 2)$. Since the value of the coupling-parameter scaling factor $\mu$ is different from that $(\mu=\alpha)$ for the zero-coupling case, the critical behavior at both ends differs from that at the zero-coupling critical point. We also note that the value of $\mu$ agrees well with that of the coupling-parameter scaling factor $(\nu=2)$ of the coupled 1D maps near both ends of each critical line segment [13]. It has been also shown in [13] that the scaling factor $\nu(=2)$ is just the only relevant $\mathrm{CE}$ of a nonzero-coupling fixed map of the renormalization transformation for the case of the coupled 1D maps.

Figure 6 shows the behavior of the asynchronous residue $R_{2, n}\left(A_{1}^{*}, c\right)$ of the synchronous orbit of period $2^{n}$ near the rightmost critical line segment in Fig. 2(a). For $c=c_{l}$ and $c_{r}$, $R_{2, n}$ converges to a critical residue $R_{2}^{*}(=0)$ as $n \rightarrow \infty$, which is different from that for the zero-coupling case. The slopes $S_{n}$ 's of $R_{2, n}$ at both ends obey well the one-term scaling law,

$$
S_{n} \sim \nu^{n} \text { for large } n
$$

The two sequences of the scaling factors $\nu_{n}$ of level $n$ at both ends are listed in Table $\mathrm{V}$, and converge to their limit values $\nu \simeq 2$, which agrees well with that of the only $\mathrm{CE}(\nu=2)$ of the nonzero-coupling fixed map governing the critical behavior at both ends for the case of the 1D maps. However, for any fixed value of $c$ inside the critical line segment, $R_{2, n}$ converges to a critical residue $R_{2}^{*}(=0.5)$ as $n \rightarrow \infty$ (see Fig. 目). This superstable case of $R_{2}^{*}=0.5$ corresponds to the supercritical case of $\lambda_{2}^{*}=0\left(\lambda_{2}^{*}\right.$ : the second critical stability multiplier) for the coupled 1D maps [13], because Eq. (223) of $R$ for the case of 2D maps reduces to the equation of $R=0.5 *(1-\lambda)$ for the case of $1 \mathrm{D}$ maps. We also note that as in the case of the coupled 1D maps, there exists no scaling factor of the coupling parameter 
inside the critical line segemnt, and hence the coupling parameter becomes an irrelevant one at interior critical points. Thus, the critical behavior inside the critical line segment becomes the same as that of the uncoupled PFDP (i.e., that of the 1D map), which will be discussed in more details below. This kind of 1D-like critical behavior was found to be governed by another nonzero-coupling fixed map with no relevant $\mathrm{CE}$ for the case of the coupled 1D maps [13].

There exists a synchronous quasiperiodic orbit on the $A=A_{1}^{*}$ line. As mentioned in Sec. [1, its synchronous Lyapunov exponents are the same as the Lyapunov exponents of the uncoupled PFDP, i.e., $\sigma_{1,1}=0$ and $\sigma_{1,2}=-0.2 \pi$. The coupling affects only the second pair of asynchronous Lyapunov exponents $\left(\sigma_{2,1}, \sigma_{2,2}\right)$, characterizing the mean exponential rate of divergence of the asynchronous mode of a nearby orbit. The maximum asynchronous Lyapunov exponent $\sigma_{2,1}$ near the rightmost critical line segment in Fig. 2(a) is shown in Fig. 5. Inside the critical line segment $\left(c_{l}<c<c_{r}\right)$, the synchronous quasiperiodic orbit on the synchronous plane becomes a synchronous attractor with $\sigma_{2,1}<0$. Since the dynamics on the synchronous attractor is the same as that of the uncoupled PFDP, the critical maps at interior points exhibit essentially 1D-like critical behaviors, because the critical behavior of the uncoupled PFDP is the same as that of the 1D maps [11]. However, as the coupling parameter $c$ passes through $c_{l}$ and $c_{r}$, the maximum asynchronous Lyapunov exponent $\sigma_{2,1}$ of the synchronous quasiperiodic orbit increases from zero. Hence, the synchronous quasiperiodic orbit becomes unstable and ceases to be an attractor outside the critical line segment. Consequently, the system of the two coupled PFDP's is asymptotically attracted to another synchronous or asynchronous attractor outside the critical line. For example, the asymptotic state for $c=0.34368\left(<c_{l}\right)$ becomes an asynchronous attractor of period 512, while that for $c=0.48479\left(>c_{r}\right)$ becomes a synchronous rotational attractor of period 1 .

We also study the critical scaling behaviors of the maximum asynchronous Lyapunov exponent $\sigma_{2,1}$ near both ends of the rightmost critical line segment in Fig. 2(a). As shown in Fig. 6, $\sigma_{2,1}$ varies linearly with respect to $c$ near both ends, i.e., $\sigma_{2,1} \sim \epsilon, \epsilon \equiv c-c^{*}\left(c^{*}=c_{l}\right.$ or $c_{r}$ ). The critical exponent of $\sigma_{2,1}$ near both ends can be also obtained from the only CE 
$\nu=2$ of the nonzero-coupling fixed map governing the critical behavior near both ends for the case of the coupled 1D maps. Consider a system with nonzero $\epsilon$ (but with $A=A_{1}^{*}$ ) near both ends. It is then transformed into a new one of the same form, but with a renormalized parameter $\epsilon^{\prime}$ under a renormalization transformation. Here the parameter $\epsilon$ obeys a scaling law,

$$
\epsilon^{\prime}=\nu \epsilon=2 \epsilon
$$

Then the maximum asynchronous Lyapunov exponent $\sigma_{2,1}$ satisfies the homogeneity relation,

$$
\sigma_{2,1}\left(\epsilon^{\prime}\right)=2 \sigma_{2,1}(\epsilon)
$$

This leads to the scaling relation,

$$
\sigma_{2,1} \sim \epsilon^{\eta}
$$

with critical exponent

$$
\eta=\ln 2 / \ln \nu=1 .
$$

As the nonlinearity parameter $A$ is further increased from $A=A_{1}^{*}$, the stationary point $\left[x_{1}=x_{2}=x^{*}=0, y_{1}=y_{2}=y^{*}=0\right]$ undergoes a cascade of "resurrections", i.e., it will restabilize after it loses its stability, destabilize again, and so ad infinitum [11]. It was found in [11] that for $\omega_{0}=0.5$, its restabilizations occur through alternating synchronous subcritical PDB's and PFB's, while the destabilizations take place via alternating synchronous supercritical PDB's and PFB's. Consequently, the two coupled PFDP's exhibit multiple period-doubling transitions to chaos. This is in contrast to the case of the coupled 1D maps, in which only single period-doubling transition to chaos occurs [12, 13].

As the first example of the multiple period-doubling transitions to chaos, we consider the first resurrection of the stationary point shown in Fig. T(a). For $A=A_{r}(1)(=3.150509 \cdots)$, a synchronous subcritical PDB occurs. Hence, the stationary point restabilizes with birth of a new unstable synchronous symmetric orbit of period 2 for $A>A_{r}(1)$. As $A$ is increased 
from $A=A_{r}(1)$, the stationary point destabilizes at $A=A_{d}(2)(=3.224230 \cdots)$ via synchronous supercritical PFB, which results in the birth of a conjugate pair of synchronous asymmetric orbits with period 1 . Fig. 0 (b) shows the stability diagram of the stationary point and the synchronous asymmetric orbits of level $n$ (period $\left.2^{n}, n=0,1,2,3,4\right)$ near the $c=0$ line in the $A-c$ plane [25]. Each synchronous asymmetric orbit of level $n$ becomes unstable at the horizontal solid line of its stable region via synchronous supercritical PDB, and gives rise to the birth of a synchronous asymmetric period-doubled orbit of level $n+1$. Such an infinite sequence terminates at a finite value of $A_{2}^{*}=3.26370315 \cdots$, which is the second period-doubling transition point of the uncoupled PFDP [11]. Note that the treelike structure of the stability diagram in Fig. O(b) is essentially the same as that in Fig. 2(a). Hence, the critical set also consists of an infinite number of critical line segments and the zero-coupling critical point, as in the first period-doubling transition case. In order to study the critical behaviors on the critical set, we follow the synchronous asymmetric orbits up to level $n=7$ in the $U$ route and the rightmost $C$ route. It is found that the critical behaviors are the same as those for the first period-doubling transition case. That is, there exist three kinds of critical behaviors at the zero-coupling critical point, both ends of each critical line segment and interior points.

As the second example, we also consider the second resurrection of the stationary point shown in Fig. 8(a). A synchronous subcritical PFB takes place at $A=A_{r}(2)$ $(=10.093985 \cdots)$. Consequently, the stationary point restabilizes with birth of a pair of new unstable orbits with period 1 . As $A$ is further increased, the stationary point destabilizes at $A=A_{d}(3)(=10.097583 \cdots)$ via synchronous supercritical PDB, which results in the birth of a new synchronous symmetric orbit with period 2. The subsequent bifurcation behaviors are the same as those for the first period-doubling transition case. That is, a third infinite sequence of synchronous supercritical PDB's follows and ends at a finite value $A_{3}^{*}$ $(=10.09966093 \cdots)$, which is the third period-doubling transition point of the uncoupled PFDP [11]. The third stability diagram of synchronous orbits near the $c=0$ line is shown in Fig. 8(b) [25]. Note that its treelike structure is essentially the same as that in Fig. 2(a). 
Hence, the critical set is composed of the zero-coupling critical point and an infinity of critical line segments. Furthermore, the critical behaviors on the critical set are found to be the same as those for the first period-doubling transition case.

In addition to the linear-coupling case (24), we have also studied other nonlinear-coupling cases,

$$
g\left(x_{1}, x_{2}\right)=\frac{c}{2}\left[x_{2}^{n}-x_{1}^{n}\right], \quad n=2,3
$$

For the first period-doubling transition case, the stability diagrams of synchronous orbits near the $c=0$ line for the cases of the quadratic and cubic couplings are shown in Fig. 9(a) and 9(b), respectively. Their treelike structures are essentially the same as that in Fig. 2(a). Hence, the zero-coupling critical point and an infinite number of critical line segments constitute the critical set for each nonlinear-coupling case. Moreover, the critical behaviors for these nonlinear-coupling cases are also found to be the same as those for the linear-coupling case.

\section{EXTENSION TO MANY COUPLED PFDP'S}

In this section we study the critical behaviors of the synchronous PDB's in $N$-coupled $(N \geq 3)$ PFDP's in which the coupling extends to the $K$ th $\left[1 \leq K \leq \frac{N}{2}\left(\frac{N-1}{2}\right)\right.$ for even (odd) $N]$ neighbor(s) with equal strength. It is found that the critical behaviors depend on the coupling range. In the global-coupling case, in which each PFDP is coupled to all the other ones with equal coupling strength, the structure of the critical set and the critical behaviors are the same as those for the two-coupled case, independently of $N$. However, for any other nonglobal-coupling cases, the structure of the critical set becomes different from that for the global-coupling case, because of a significant change in the stability diagram.

Consider $N$ symmetrically coupled PFDP's with a periodic boundary condition,

$$
\ddot{x}_{m}=f\left(x_{m}, \dot{x}_{m}, t\right)+g\left(x_{m}, x_{m+1}, \ldots, x_{m-1}\right), \quad m=1,2, \ldots, N \text {. }
$$


Here the periodic boundary condition imposes $x_{m}(t)=x_{m+N}(t)$ for all $m$, the function $f(x, \dot{x}, t)$ is given in Eq. (2), and $g\left(x_{1}, \ldots, x_{N}\right)$ is a coupling function, obeying the condition

$$
g(x, \ldots, x)=0 \text { for all } x .
$$

A general form of coupling for odd $N(N \geq 3)$ is given by

$$
\begin{aligned}
g\left(x_{1}, \ldots, x_{N}\right)= & \frac{c}{2 K+1} \sum_{l=-K}^{K}\left[u\left(x_{1+l}\right)-u\left(x_{1}\right)\right], \\
= & c\left[\frac{1}{2 K+1} \sum_{l=-K}^{K} u\left(x_{1+l}\right)-u\left(x_{1}\right)\right], \\
& K=1, \ldots, \frac{N-1}{2},
\end{aligned}
$$

where $c$ is a coupling parameter and $u$ is a function of one variable. Here the coupling extends to the $K$ th neighbors with equal coupling strength, and the function $g$ satisfies the condition (46). The extreme long-range interaction for $K=\frac{N-1}{2}$ is called a global coupling, for which the coupling function $g$ becomes

$$
\begin{aligned}
g\left(x_{1}, \ldots, x_{N}\right) & =\frac{c}{N} \sum_{m=1}^{N}\left[u\left(x_{m}\right)-u\left(x_{1}\right)\right] \\
& =c\left[\frac{1}{N} \sum_{m=1}^{N} u\left(x_{m}\right)-u\left(x_{1}\right)\right] .
\end{aligned}
$$

This is a kind of mean-field coupling, in which each element is coupled to all the other elements with equal coupling strength. All the other couplings with $K<\frac{N-1}{2}$ (e.g., nearestneighbor coupling with $K=1$ ) will be referred to as non-global couplings. The $K=1$ case for $N=3$ corresponds to both the global coupling and the nearest-neighbor coupling.

We next consider the case of even $N(N \geq 2)$. The form of coupling of Eq. (47) holds for the cases of non-global couplings with $K=1, \ldots, \frac{N-2}{2}(N \geq 4)$. The global coupling for $K=\frac{N}{2}(N \geq 2)$ also has the form of Eq. (48), but it cannot have the form of Eq. (47), because there exists only one farthest neighbor for $K=\frac{N}{2}$, unlike the case of odd $N$. The $K=1$ case for $N=2$ also corresponds to the nearest-neighbor coupling as well as to the global coupling, like the $N=3$ case. 
The stability analysis of an orbit in many coupled PFDP's is conveniently carried out by Fourier-transforming with respect to the discrete space $\{m\}$ [26]. Consider an orbit $\left\{x_{m}(t) ; m=1, \ldots, N\right\}$ of the $N$ coupled PFDP's (45). The discrete spatial Fourier transform of the orbit is:

$$
\begin{gathered}
\mathcal{F}\left[x_{m}(t)\right] \equiv \frac{1}{N} \sum_{m=1}^{N} e^{-2 \pi i m j / N} x_{m}(t)=\xi_{j}(t), \\
j=0,1, \ldots, N-1 .
\end{gathered}
$$

The Fourier transform $\xi_{j}(t)$ satisfies $\xi_{j}^{*}(t)=\xi_{N-j}(t)$ (* denotes complex conjugate), and the wavelength of a mode with index $j$ is $\frac{N}{j}$ for $j \leq \frac{N}{2}$ and $\frac{N}{N-j}$ for $j>\frac{N}{2}$.

To determine the stability of a synchronous $q$-periodic orbit $\left[x_{1}(t)=\cdots=x_{N}(t) \equiv x^{*}(t)\right.$ for all $t$ and $x^{*}(t)=x^{*}(t+q)$ ], we consider an infinitesimal perturbation $\left\{\delta x_{m}(t)\right\}$ to the synchronous orbit, i.e., $x_{m}(t)=x^{*}(t)+\delta x_{m}(t)$ for $m=1, \ldots, N$. Linearizing the $N$-coupled PFDP's (45) at the synchronous orbit, we obtain:

$$
\begin{aligned}
\delta \ddot{x}_{m}= & \frac{\partial f\left(x^{*}, \dot{x}^{*}, t\right)}{\partial x^{*}} \delta x_{m}+\frac{\partial f\left(x^{*}, \dot{x}^{*}, t\right)}{\partial \dot{x}^{*}} \delta \dot{x}_{m} \\
& +\sum_{l=1}^{N} G_{l}\left(x^{*}\right) \delta x_{l+m-1},
\end{aligned}
$$

where

$$
\left.G_{l}(x) \equiv \frac{\partial g\left(x_{1}, \ldots, x_{N}\right)}{\partial x_{l}}\right|_{x_{1}=\cdots=x_{N}=x} .
$$

Hereafter the functions $G_{l}$ 's will be called "reduced" coupling functions of $g\left(x_{1}, \ldots, x_{N}\right)$.

Let $\delta \xi_{j}(t)$ be the Fourier transform of $\delta x_{m}(t)$, i.e.,

$$
\begin{aligned}
\delta \xi_{j}=\mathcal{F}\left[\delta x_{m}(t)\right]=\frac{1}{N} \sum_{m=1}^{N} e^{-2 \pi i m j / N} \delta x_{m}, \\
j=0,1, \ldots, N-1 .
\end{aligned}
$$

Here $\delta \xi_{0}$ is the synchronous-mode perturbation, and all the other $\delta \xi_{j}$ 's with nonzero indices $j$ are the asynchronous-mode perturbations. Then the Fourier transform of Eq. (50) becomes:

$$
\begin{aligned}
\delta \ddot{\xi}_{j}= & \frac{\partial f\left(x^{*}, \dot{x}^{*}, t\right)}{\partial \dot{x}^{*}} \delta \dot{\xi}_{j}+\left[\frac{\partial f\left(x^{*}, \dot{x}^{*}, t\right)}{\partial x^{*}}\right. \\
& \left.+\sum_{l=1}^{N} G_{l}\left(x^{*}\right) e^{2 \pi i(l-1) j / N}\right] \delta \xi_{j}, \quad j=0,1, \ldots, N-1 .
\end{aligned}
$$


Note that all the modes $\delta \xi_{j}$ 's become decoupled for the synchronous orbit.

The equation (53) can also be put into the following form:

$$
\left(\begin{array}{c}
\delta \dot{\xi}_{j} \\
\delta \dot{\eta}_{j}
\end{array}\right)=L_{j}(t)\left(\begin{array}{c}
\delta \xi_{j} \\
\delta \eta_{j}
\end{array}\right), j=0,1, \ldots, N-1,
$$

where

$$
L_{j}(t)=\left(\begin{array}{cc}
0 & 1 \\
\frac{\partial f\left(x^{*}, \dot{x}^{*}, t\right)}{\partial x^{*}}+\sum_{l=1}^{N} G_{l}\left(x^{*}\right) e^{2 \pi i(l-1) j / N} & \frac{\partial f\left(x^{*}, \dot{x}^{*}, t\right)}{\partial \dot{x}^{*}}
\end{array}\right) .
$$

Note that each $L_{j}$ is a $q$-periodic matrix, i.e., $L_{j}(t)=L_{j}(t+q)$. Let $\Phi_{j}(t)=\left(\phi_{j}^{(1)}(t), \phi_{j}^{(2)}(t)\right)$ be a fundamental solution matrix with $\Phi_{j}(0)=I$. Here $\phi_{j}^{(1)}(t)$ and $\phi_{j}^{(2)}(t)$ are two independent solutions expressed in column vector forms, and $I$ is the $2 \times 2$ unit matrix. Then a general solution of the $q$-periodic system has the following form

$$
\begin{aligned}
\left(\begin{array}{c}
\delta \xi_{j}(t) \\
\delta \eta_{j}(t)
\end{array}\right)=\Phi_{j}(t) & \left(\begin{array}{c}
\delta \xi_{j}(0) \\
\delta \eta_{j}(0)
\end{array}\right), \\
& j=0,1, \ldots, N-1,
\end{aligned}
$$

Substitution of Eq. (56) into Eq. (54) leads to an initial-value problem to determine $\Phi_{j}(t)$,

$$
\dot{\Phi}_{j}(t)=L_{j}(t) \Phi_{j}(t), \Phi_{j}(0)=I
$$

Each $2 \times 2$ matrix $\Psi_{j}\left[\equiv \Phi_{j}(q)\right]$, which is obtained through integration of Eq. (57) over the period $q$, determines the stability of the q-periodic synchronous orbit against the $j$ th-mode perturbation.

The characteristic equation of each matrix $\Psi_{j}(j=0,1, \ldots, N-1)$ is

$$
\lambda_{j}^{2}-\operatorname{tr} \Psi_{j} \lambda_{j}+\operatorname{det} \Psi_{J}=0
$$

where $\operatorname{tr} \Psi_{j}$ and $\operatorname{det} \Psi_{j}$ denote the trace and determinant of $\Psi_{j}$, respectively. As shown in [17], $\operatorname{det} \Psi_{j}$ is given by

$$
\operatorname{det} \Psi_{j}=e^{\int_{0}^{q} \operatorname{tr} L_{j} d t}=e^{-2 \pi \gamma q}
$$


Hence, all the matrices $\Psi_{j}$ 's have the same constant Jacobian determinant (less than unity). The eigenvalues, $\lambda_{j, 1}$ and $\lambda_{j, 2}$, of $\Psi_{j}$ are called the Floquet stability multipliers, which are associated with the stability of the synchronous $q$-periodic orbit against the $j$ th-mode perturbation. Since the $j=0$ case corresponds to the synchronous mode, the first pair of stability multipliers $\left(\lambda_{0,1}, \lambda_{0,2}\right)$ is called the pair of synchronous stability multipliers. On the other hand, all the other pairs of stability multipliers are called the pairs of asynchronous stability multiplies, because all the other cases of $j \neq 0$ correspond to asynchronous modes. Like the two-coupled case [see Eq. (23)], we also associate with a pair of stability multipliers $\lambda_{j, 1}$ and $\lambda_{j, 2}$ a residue $R_{j}$,

$$
R_{j} \equiv \frac{1+\operatorname{det} \Psi_{j}-\operatorname{tr} \Psi_{j}}{2\left(1+\operatorname{det} \Psi_{j}\right)}, \quad j=0,1, \ldots, N-1 .
$$

Here the first one $R_{0}$ is associated with the stability against the synchronous-mode perturbation, and hence it may be called the synchronous residue. On the other hand, all the other ones $R_{j}(j \neq 0)$ are called the asynchronous residues, because they are associated with the stability against the asynchronous-mode perturbations.

It follows from the condition (46) that the reduced coupling functions satisfy

$$
\sum_{l=1}^{N} G_{l}(x)=0 .
$$

Hence the matrix (55) for $j=0$ becomes

$$
L_{0}(t)=\left(\begin{array}{cc}
0 & 1 \\
\frac{\partial f\left(x^{*}, \dot{x}^{*}, t\right)}{\partial x^{*}} & \frac{\partial f\left(x^{*}, \dot{x}^{*}, t\right)}{\partial \dot{x}^{*}}
\end{array}\right) .
$$

This is just the linearized Poincaré map of the uncoupled PFDP [11]. Hence the synchronous residue $R_{0}$ becomes the same as the residue of the uncoupled PFDP, i.e., it depends only on the nonlinearity parameter $A$. While there is no coupling effect on $R_{0}$, the coupling affects all the other asynchronous residues $R_{j}(j \neq 0)$.

In case of the global coupling of Eq. (48), the reduced coupling functions become:

$$
G_{l}(x)=\left\{\begin{array}{cc}
(1-N) G(x) & \text { for } l=1 \\
G(x) & \text { for } l \neq 1
\end{array}\right.
$$


where $G(x)=\frac{c}{N} u^{\prime}(x)$. Substituting $G_{l}$ 's into the second term of the $(2,1)$ entry of the matrix $L_{j}(t)$, we have:

$$
\sum_{l=1}^{N} G_{l}(x) e^{2 \pi i(l-1) j / N}=\left\{\begin{array}{cc}
0 & \text { for } j=0 \\
-c u^{\prime}(x) & \text { for } j \neq 0
\end{array}\right.
$$

Hence all the asynchronous residues $R_{j}(j \neq 0)$ become the same, i.e., $R_{1}=\cdots=R_{N-1}$. Consequently, like the two-coupled case, there exist only two independent residues $R_{0}$ and $R_{1}$, the values of which are also independent of $N$.

We next consider the non-global coupling of the form (47) and define

$$
G(x) \equiv \frac{c}{2 K+1} u^{\prime}(x)
$$

where $1 \leq K \leq \frac{N-2}{2}\left(\frac{N-3}{2}\right)$ for even (odd) $N$ larger than 3 . Then we have

$$
G_{l}(x)=\left\{\begin{array}{cl}
-2 K G(x) & \text { for } l=1 \\
G(x) & \text { for } 2 \leq l \leq 1+K \text { or } \\
& \text { for } N+1-K \leq l \leq N \\
0 & \text { otherwise. }
\end{array}\right.
$$

Substituting the reduced coupling functions into the matrix $L_{j}(t)$ of Eq. (55), the second term of the $(2,1)$ entry of $L_{j}(t)$ becomes:

$$
\sum_{l=1}^{N} G_{l}(x) e^{2 \pi i(l-1) j / N}=-S_{N}(K, j) c u^{\prime}(x),
$$

where

$$
S_{N}(K, j) \equiv \frac{4}{2 K+1} \sum_{k=1}^{K} \sin ^{2} \frac{\pi j k}{N}=1-\frac{\sin (2 K+1) \frac{\pi j}{N}}{(2 K+1) \sin \frac{\pi j}{N}}
$$

Hence, unlike the global-coupling case, all the asynchronous residues vary depending on the coupling range $K$ as well as on the mode number $j$. Since $S_{N}(K, j)=S_{N}(K, N-j)$, the residues satisfy

$$
R_{j}=R_{N-j}, \quad j=0,1, \ldots, N-1
$$


Thus it is sufficient to consider only the case of $0 \leq j \leq \frac{N}{2}\left(\frac{N-1}{2}\right)$ for even (odd) $N$. Comparing the expression in Eq. (67) with that in Eq. (64) for $j \neq 0$, one can easily see that they are the same except for the factor $S_{N}(K, j)$. Consequently, making a change of the coupling parameter $c \rightarrow \frac{c}{S_{N}(K, j)}$, the residue $R_{j}$ for the non-global coupling case of range $K$ becomes the same as that for the global-coupling case.

Each pair of stability multipliers $\left(\lambda_{j, 1}, \lambda_{j, 2}\right)(j=0,1, \ldots, N-1)$ lies either on the circle of radius $e^{-\pi \gamma q}$, or on the real axis in the complex plane. The synchronous orbit is stable against the $j$ th-mode perturbation when $0<R_{j}<1$ (i.e., the pair of stability multipliers $\left(\lambda_{j, 1}, \lambda_{j, 2}\right)$ lies inside the unit circle in the complex plane). A PDB (PFB) occurs when the residue $R_{j}$ increases (decreases) through 1 (0) [i.e., a stability multiplier decreases (increases) through $-1(1)$ ]. We also note that a(n) synchronous (asynchronous) bifurcation takes place for $j=0(j \neq 0)$. For more details on bifurcatios, refer to Sec. II].

When the synchronous residue $R_{0}$ of a synchronous periodic orbit increases through 1 , the synchronous orbit loses its stability via synchronous PDB, giving rise to the birth of a new synchronous period-doubled orbit. Here we are interested in such synchronous PDB's. Thus, for each mode with nonzero index $j$ we consider a region in the $A-c$ plane, in which the synchronous orbit is stable against the perturbations of both modes with indices 0 and $j$. This stable region is bounded by four bifurcation curves determined by the equations $R_{0}=0,1$ and $R_{j}=0,1$, and it will be denoted by $U_{N}$.

For the case of global coupling, those stable regions coincide, irrespectively of $N$ and $j$, because all the asynchronous residues $R_{j}$ 's $(j \neq 0)$ are the same, independently of $N$. The stable region for this global-coupling case will be denoted by $U_{G}$. Note that $U_{G}$ itself is just the stability region of the synchronous orbit, irrespectively of $N$, because the synchronous orbit is stable against the perturbations of all synchronous and asynchronous modes in the region $U_{G}$. Thus the stability diagram of synchronous orbits of period $2^{n}(n=1,2,3, \ldots)$ in the $A-c$ plane becomes the same as that for the two-coupled case, independently of $N$. That is, the stable regions of the synchronous orbits form a "stability tree" in the parameter 
plane [see Figs. 2(a) and 2(b)]. Consequently, the zero-coupling critical point and an infinite number of critical line segments constitute the critical set. There exists one kind of critical behavior in the $U$ route ending at the zero-coupling critical point, while two other kinds of critical behaviors exist in each $C$ route ending at a critical line segment. The three kinds of critical behaviors are the same as those for the two-coupled case, independently of $N$. For more details on the critical behaviors, refer to Sec. III.

However, the stable region $U_{N}$ vary depending on the coupling range $K$ and the mode number $j$ for the nonglobal-coupling cases, i.e., $U_{N}=U_{N}(K, j)$. To find the stability region of a synchronous orbit in $N$ coupled PFDP's with a given $K$, one may start with the stability region $U_{G}$ for the global-coupling case. Rescaling the coupling parameter $c$ by a scaling factor $\frac{1}{S_{N}(K, j)}$ for each nonzero $j$, the stable region $U_{G}$ is transformed into a stable region $U_{N}(K, j)$. Then the stability region of the synchronous orbit is given by the intersection of all such stable regions $U_{N}$ 's. An important change occurs in the stability diagram of the synchronous orbits of period $2^{n}(n=1,2, \ldots)$, and consequently the structure of the critical set becomes different from that for the global-coupling case, as will be seen below.

As an example, we consider the nearest-neighbor coupling case with $K=1$ in four linearly-coupled PFDP's, in which the coupling function is given by

$$
g\left(x_{1}, x_{2}, x_{3}, x_{4}\right)=\frac{c}{3}\left(x_{2}+x_{4}-2 x_{1}\right)
$$

Figure 10 shows the stability regions of the synchronous $2^{n}$-periodic $(n=1,2,3,4)$ orbits. Note that the scaling factor $\frac{1}{S_{4}(1, j)}$ has its minimum value $\frac{3}{4}$ at $j=2$. However, for each synchronous orbit, $U_{4}(1,2)$ itself cannot be the stability region, because bifurcation curves of different modes with nonzero indices intersect one another. We first examine the structure of the stability diagram in Fig. 10(a), starting from the left side of the stability region of the synchronous orbit of level $1(n=1)$. The zero $c$ side of $U_{4}(1,2)$ including a $c=0$ line segment remains unchanged, whereas the other side becomes flattened by the bifurcation curve of the asynchronous mode with $j=1$. Due to the successive flattening with increasing level $n$, a significant change in the stability diagram occurs. Of the infinite number of period- 
doubling routes for the global-coupling case, only the $U$ route ending at the zero-coupling critical point remains. Thus only the zero-coupling point is left as a critical point in the parameter plane. However, as shown in Fig. $10(\mathrm{~b})$, the rightmost branch of the stability diagram, starting from the right side of the stability region of the synchronous periodic orbit of level 1 , is the same as that for the global-coupling case except that the coupling parameter $c$ is rescaled with the maximum scaling factor $\frac{1}{S_{4}(1,1)}(=1.5)$ of the $j=1$ mode. Hence, the rightmost $C$ route ending at a critical line segment is also left. Consequently, the critical set for this linear-coupling case is composed of the zero-coupling critical point and one critical line segment.

Consider a self-similar sequence of parameters $\left(A_{n}, c_{n}\right)$, at which the synchronous orbits of period $2^{n}$ has some given residues, in the $U$ route for the global-coupling case. Rescaling the coupling parameter with the minimum scaling factor $S_{4}(1,2)(=0.75)$, the sequence is transformed into a self-similar one for the $N=4$ case of nearest-neighbor coupling. Hence, the critical behavior near the zero-coupling critical point becomes the same as that for the global-coupling case. As mentioned above, the rightmost $C$ route in Fig. 2(b) for the globalcoupling case is also transformed into the $C$ route in Fig. 10(b) for the nearest-neighbor coupling case by rescaling $c$ with the maximum scaling factor $S_{4}(1,1)(=1.5)$. Hence, the critical behaviors at both ends and interior points of the critical line segment are the same as those for the global-coupling case.

The results for the nearest-neighbor coupling case with $K=1$ extends to all the other nonglobal-coupling cases with $1<K<\frac{N}{2}\left(\frac{N-1}{2}\right)$ for even (odd) $N$. For each nonglobalcoupling case with $K>1$, we first consider a mode with index $j_{\min }$ for which the scaling factor $\frac{1}{S_{N}(K, j)}$ becomes the smallest one and the stability region $U_{N}\left(K, j_{\min }\right)$ including a $c=0$ line segment. Here the value of $j_{\min }$ varies depending on the range $K$. Like the $K=1$ case, the zero $c$ side of $U_{N}\left(K, j_{\text {min }}\right)$ including the $c=0$ line segemnt remains unchanged, whereas the other side becomes flattened by the bifurcation curves of the other modes with nonzero indices. Thus the overall shape of the stability diagram, starting from the left zero $c$ side of the stability region of the synchronous 2-periodic orbit, becomes essentially the same as 
that for the nearest-neighbor coupling case. Consequently, only the $U$ route ending at the zero-coupling critical point is left as a period-doubling route, and the critical behavior near the zero-coupling critical point is also the same as that for the global-coupling case. We next consider a mode with index $j_{\max }$ for which the scaling factor $\frac{1}{S_{N}(K, j)}$ becomes the largest one. Rescaling $c$ with the maximum scaling factor $\frac{1}{S_{N}\left(K, j_{\max }\right)}$, the rightmost $C$ route in Fig. 2(b) for the global-coupling case is transformed into the $C$ route for the nonglobal-coupling case, and the critical behaviors at the critical line segment are also the same as those for the global-coupling case.

\section{SUMMARY}

The critical behaviors of PDB's in $N$ coupled PFDP's are investigated by varying two parameters $A$ and $c$. As $A$ is increased, the stationary point of the coupled PFDP's undergoes an infinite series of period-doubling transitions to chaos. This is in contrast to the case of the coupled 1D maps with only single period-doubling transition to chaos [12,13. The twocoupled case with $N=2$ has been first studied. For each period-doubling transition to chaos, the zero-coupling critical point and an infinity of critical line segments constitute the critical set in the parameter plane. There are three kinds of critical behaviors, depending on the position of the critical set. They are found to be the same as those for the coupled 1D maps [13]. We also extend the results of the two-coupled case to many coupled PFDP's, in which the critical behaviors vary depending on whether or not the coupling is global. In the global-coupling case, the critical behaviors are the same as those for the two-coupled case, independently of $N$. However, for any other nonglobal-coupling cases, the structure of the critical set becomes different from that for the global-coupling case, because of an important change in the stability diagram of $2^{n}$-periodic orbits $(n=0,1,2, \ldots)$. 


\section{ACKNOWLEDGMENTS}

This work was supported by the Exchange Program of the Senior Scientist, the Korea Science and Engineering Foundation. One of us (S.Y.K.) thanks Professor R. Fox and

Ms. M. Choi for their hospitality during the period of his visit to the Georgia Institute of Technology. 


\section{REFERENCES}

[1] R.V. Buskirk and C. Jeffries, Phys. Rev. A 31, 3332 (1985).

[2] P. Hadley and M.R. Beasley, Appl. Phys. Lett. 50, 621 (1987); P. Hadley, M.R. Beasley, and K. Wiesenfeld, Phys. Rev. B 38, 8712 (1988).

[3] S.H. Strogatz, C.M. Marcus, R.M. Westervelt, and R.E. Mirollo, Physica D 36, 23 (1989).

[4] Y. Kuramoto, Chemical Oscillations, Waves and Turbulence (Springer-Verlag, New York, 1984).

[5] A.T. Winfree, The Geometry of Biological Time (Springer-Verlag, Berlin, 1980).

[6] L.D. Landau and E.M. Lifshitz, Mechanics (Pergamon Press, New York, 1976), p. 80.

[7] V.I. Arnold, Mathematical Methods of Classical Mechanics (Springer-Verlag, New York, 1978), p. 113; Ordinary Differential Equations (MIT press, Cambridge, 1973), p. 203.

[8] J.B. McLaughlin, J. Stat. Phys. 24, 375 (1981).

[9] R.W. Leven and B.P. Koch, Phys. Lett. A 86, 71 (1981); B.P. Koch, R.W. Leven, B. Pompe, and C. Wilke, ibid. 96, 219 (1983); B.P. Koch and R.W. Leven, Physica D 16, 1 (1985); R.W. Leven, B. Pompe, C. Wilke, and B.P. Koch, ibid. 16, 371 (1985).

[10] A. Arneodo, P. Coullet, C. Tresser, A. Libchaber, J. Maurer, and D. d'Humières, Physica D 6, 385 (1983).

[11] S.-Y. Kim and K. Lee, Phys. Rev. E 53, 1579 (1996).

[12] S. Kuznetsov, Radiophys. Quantum Electron. 28, 681 (1985); H. Kook, F.H. Ling, and G. Schmidt, Phys. Rev. A 43, 2700 (1991).

[13] S.-Y. Kim and H. Kook, Phys. Rev. A 46, R4467 (1992); Phys. Lett. A 178, 258 (1993); Phys. Rev. E 48, 785 (1993); in the Proceeding of the First International Workshop on 
Nonlinear Dynamics and Chaos, edited by H. Lee (Pohang Institute of Science and Technology, Pohang, Korea, 1993), pp. 49-90.

[14] F.H. Ling, G. Schmidt, and H. Kook, Int. J. Bif. Chaos 1, 363 (1991).

[15] Without giving any explicit numerical values of the scaling factors, the authors of the paper [14] insisted that the critical behaviors of the coupled oscillators are the same as those of the coupled 1D maps, based on the stability diagram of orbits with only period 2 and 4 (see Figs. 1 and 2 in [14]).

[16] S. Lefschetz, Differential Equations: Geometric Theory (Dover Publications, Inc., New York, 1977), Sec. 3.5.

[17] S. Lefschetz, Differential Equations: Geometric Theory (Dover Publications, Inc., New York, 1977), p. 60.

[18] S.-Y. Kim and B. Hu, Phys. Rev. A 44, 934 (1991); S.-Y. Kim and D.-S. Lee, Phys. Rev. A 45, 5480 (1992).

[19] J. Gukenheimer and P. Holmes, Nonlinear Oscillations, Dynamical Systems, and Bifurcations of Vector Fields (Springer-Verlag, New York, 1983), Sec. 3.5.

[20] A.J. Lichtenberg and M.A. Lieberman, Regular and Stochastic Motion (Springer-Verlag, New York, 1983), Sec. 5.3.

[21] M.J. Feigenbaum, J. Stat. Phys. 19, 25 (1978); 21, 669 (1979).

[22] S.-Y. Kim, Phys. Rev. E 49, 1745 (1994).

[23] S.-Y. Kim, Phys. Rev. E 50, 4237 (1994).

[24] J.-m Mao and B. Hu, J. Stat. Phys. 46, 111 (1987); Int. J. Mod. Phys. B 2, 65 (1988); C. Reick, Phys. Rev. A 45, 777 (1992).

[25] Unlike the case of the first period-doubling transition to chaos with only one "stability 
tree" consisting of connected stability regions of synchronous orbits of all levels, several separate stability trees are found in the $A-c$ plane for the case of the second (third) period-doubling transition to chaos. Only the stability tree starting from the stability region of the stationary point including the $c=0$ line is shown in Fig. Q 7 (b) [8(b)], whose structure is essentially the same as that in Fig. 2(a). However, other separate stability trees grow like chimneys without $U$-shape branchings, as in Fig. 2(b), where the critical behaviors are also the same as those in the $C$-route for the first perioddoubling transition case.

[26] I. Waller and R. Kapral, Phys. Rev. A 30, 2047 (1984). 


\section{TABLES}

TABLE I. In the $U$ route, we followed a sequence of parameters $\left(A_{n}, c_{n}\right)$ at which the pair of residues $\left(R_{1, n}, R_{2, n}\right)$ of the synchronous orbit of period $2^{n}$ is $(1,0)$. This sequence converges to the zero-coupling critical point $\left(A_{1}^{*}, 0\right)$. The scaling factors of the nonlinearity and coupling parameters $A$ and $c$ are shown in the second and third columns, respectively.

\begin{tabular}{lcr}
\hline \hline$n$ & $\delta_{n}$ & $\mu_{n}$ \\
\hline 2 & 5.286 & -2.96 \\
3 & 4.692 & -2.91 \\
4 & 4.665 & -2.41 \\
5 & 4.666 & -2.59 \\
6 & 4.667 & -2.43 \\
7 & 4.670 & -2.57 \\
8 & 4.665 & -2.45 \\
\hline \hline
\end{tabular}

TABLE II. For the case of the $U$ route, the scaling factors $\mu_{1, n}$ and $\mu_{2, n}$ in the two-term scaling for the coupling parameter are shown in the second and third columns, respectively. A product of them, $\frac{\mu_{1, n}^{2}}{\mu_{2, n}}$, is shown in the fourth column.

\begin{tabular}{lrrr}
\hline \hline$n$ & $\mu_{1, n}$ & $\mu_{2, n}$ & $\frac{\mu_{1, n}^{2}}{\mu_{2, n}}$ \\
\hline 4 & -2.536 & 6.87 & 0.94 \\
5 & -2.500 & 2.84 & 2.20 \\
6 & -2.500 & 2.81 & 2.22 \\
7 & -2.504 & 3.09 & 2.03 \\
\hline \hline
\end{tabular}


TABLE III. The scaling factors $\nu_{1, n}$ and $\nu_{2, n}$ in the two-term scaling for the slope $S_{n}$ of the asynchronous residue $R_{2, n}$ at the zero-coupling critical point are shown in the second and third columns, respectively.

\begin{tabular}{lcr}
\hline \hline$n$ & $\nu_{1, n}$ & $\nu_{2, n}$ \\
\hline 4 & -2.599 & 2.783 \\
5 & -2.511 & 1.923 \\
6 & -2.503 & 2.004 \\
7 & -2.503 & 1.998 \\
8 & -2.503 & 1.999 \\
\hline \hline
\end{tabular}

TABLE IV. We followed, in the rightmost $C$ route in Fig. 2(a), two self-similar sequences of parameters $\left(A_{n}, c_{n}\right)$, at which the pair of residues $\left(R_{1, n}, R_{2, n}\right)$ of the synchronous orbit with period $2^{n}$ is $(1,0.1)$. They converge to both ends of the critical line segment. The scaling factors of the coupling paramter at the left and right ends are shown in the second and third columns, respectively. In both cases the scaling factors seem to converge to the same limit value $\mu \simeq 2$.

\begin{tabular}{lcc}
\hline \hline$n$ & $\mu_{n}$ & $\mu_{n}$ \\
\hline 5 & 1.05 & 3.12 \\
6 & 1.76 & 2.55 \\
7 & 1.85 & 2.26 \\
8 & 1.94 & 2.12 \\
\hline \hline
\end{tabular}


TABLE V. The scaling factors $\nu_{n}$ 's in the one-term scaling for the slopes $S_{n}$ 's of the asynchronous residue $R_{2, n}$ at the left and right ends of the rightmost critical line segment in Fig. 2(a) are shown in the second and third columns, respectively.

\begin{tabular}{lcc}
\hline \hline$n$ & $\nu_{n}$ & $\nu_{n}$ \\
\hline 4 & 2.156 & 1.991 \\
5 & 1.971 & 2.003 \\
6 & 2.006 & 1.999 \\
7 & 1.999 & 2.000 \\
8 & 2.000 & 2.000 \\
\hline \hline
\end{tabular}




\section{FIGURES}

FIG. 1. Stability diagram of the synchronous orbits of low period $q=1,2$ in two linearly coupled PFDP's. The stable regions of the stationary point, a symmetric 2-periodic orbit, and an asymmetric 2-periodic orbit are denoted by SP, SP2, and ASP2, respectively. The horizontal (non-horizontal) solid and short-dashed boundary lines correspond to synchronous (asynchronous) PDB and PFB lines, respectively. For other details see the text.

FIG. 2. Stability diagram of synchronous asymmetric $2^{n}$-periodic $(n=1,2,3,4,5)$ orbits of level $n$ born via synchronous supercritical PDB's. ASP2 denotes the stable region of an asymmetric orbit of level 1, and PN also designates the stable region of an asymmetric orbit of period $\mathrm{N}$ $(\mathrm{N}=4,8,16,32)$. The solid and short-dashed boundary lines represent the same as those in Fig. 1. The stability diagram starting from the left (right) side of the ASP2 is shown in (a) [(b)]. Note its treelike structure. See the text for other details.

FIG. 3. Plots of the asynchronous residue $R_{2, n}\left(A_{1}^{*}, c\right)$ versus $c$ near the zero-coupling critical point for $n=4,5,6$.

FIG. 4. Plots of the asynchronous residue $R_{2, n}\left(A_{1}^{*}, c\right)$ versus $c$ near the rightmost critical line in Fig. 2(a) for $n=5,6,7$.

FIG. 5. Maximum asynchronous Lyapunov exponent $\sigma_{2,1}$ of the synchronous quasiperiodic orbit near the rightmost critical line in Fig. 2(a). The values of $\sigma_{2,1}$ at both ends of the rightmost critical line are zero, which are denoted by solid circles.

FIG. 6. Maximum asynchronous Lyapunov exponents $\sigma_{2,1}$ of the synchronous quasiperiodic orbit near both (a) the left end and (b) the right end of the rightmost critical line in Fig. 2(a). Here $\epsilon=c-c^{*}\left(c^{*}=c_{l}\right.$ or $\left.c_{r}\right)$. Note that $\sigma_{2,1}$ varies linearly with respect to $c$ near both ends. 
FIG. 7. (a) Bifurcation diagram (plot of $x^{*}$ versus $A$ ) in the vicinity of the first resurrection of the stationary point with $x^{*}=0 ; x_{1}=x_{2} \equiv x^{*}$ for a synchronous orbit. Here $q=1(2)$ denotes the period of a synchronous orbit, born via supercritical PFB (subcritical PDB). The solid and short-dashed lines also designate stable and unstable orbits, respectively. (b) Second stability diagram of synchronous orbits near the $c=0$ line. Here SP, ASP1, and PN denote the stable regions of the stationary point, an asymmetric orbit of period 1, and an asymmetric N-periodic $(\mathrm{N}=2,4,8,16)$ orbit, respectively. The solid and short-dashed boundary lines also represent the same as those in Fig. 1. For other details see the text.

FIG. 8. (a) Bifurcation diagram (plot of $x^{*}$ versus $A$ ) in the vicinity of the second resurrection of the stationary point with $x^{*}=0 ; x_{1}=x_{2} \equiv x^{*}$ for a synchronous orbit. Here $q=1(2)$ denotes the period of a synchronous orbit, born via subcritical PFB (supercritical PDB). As in Fig. 7(a), the solid and short-dashed lines also designate stable and unstable orbits, respectively. (b) Third stability diagram of synchronous orbits near the $c=0$ line. Here SP, SP2, ASP2, and PN $(\mathrm{N}=4,8,16)$ denote the stable regions of the stationary point, a symmetric orbit of period 2, an asymmetric 2-periodic orbit, and an asymmetric orbit with period $\mathrm{N}(\mathrm{N}=4,8,16)$ orbit, respectively. The solid and short-dashed boundary lines also represent the same as those in Fig. 1. For other details see the text.

FIG. 9. Stability diagrams of synchronous orbits near the $c=0$ line for the cases of (a) the quadratic and (b) cubic couplings. Here SP2, ASP2, and PN $(\mathrm{N}=4,8)$ denote the stable regions of a symmetric orbit of period 2, an asymmetric 2-periodic orbit, and an asymmetric orbit with period $\mathrm{N}$, respectively.

FIG. 10. Stability diagram of synchronous orbits in four linearly-coupled PFDP's. Each stable region is bounded by its solid boundary curves. For a synchronous orbit of period $q$, the $\mathrm{PDB}(\mathrm{PFB})$ curve of the mode with index $j$ is denoted by a symbol $q_{j}^{P D(P F)}$. The stability diagram starting from the left (right) side of a 2-periodic orbit is shown in (a) [(b)]. For other details see the text. 